TRANSACTIONS OF THE

AMERICAN MATHEMATICAL SOCIETY

Volume 354, Number 9, Pages 3771-3787

S 0002-9947(02)02972-0

Article electronically published on April 22, 2002

\title{
SUBMERSIONS, FIBRATIONS AND BUNDLES
}

\author{
GAËL MEIGNIEZ
}

\begin{abstract}
When does a submersion have the homotopy lifting property? When is it a locally trivial fibre bundle? We establish characterizations in terms of consistency in the topology of the neighbouring fibres.
\end{abstract}

In differential topology, one meets nonproper submersive maps, and hopes that they will be fibrations (resp. fibre bundles) under hypotheses of consistency between the homotopy type (resp. topology) of the neighbouring fibres. The aim of this paper is to give suitable characterizations.

\section{Submersions And Fibrations}

This first part of this paper belongs to the most elementary homotopy theory. Our purpose is to establish the following homotopy lifting characterization, and a few corollaries.

Theorem A. A surjective map is a fibration if and only if it satisfies the following three conditions: it is a homotopic submersion, all vanishing cycles of all dimensions are trivial, and all emerging cycles of all dimensions are trivial.

Let us first specify definitions, conventions and notations.

I.1. Definitions. Throughout this paper, "space" means Hausdorff topological space, "map" means continuous map, "polytope" means finite simplicial complex. For every $p \geq 0$, denote by $\mathbf{B}^{p}$ the compact $p$-ball and $\mathbf{S}^{p}=\partial \mathbf{B}^{p+1}$ the $p$-sphere. Fix a basepoint $* \in \mathbf{S}^{p}$.

Let $E, B$ be two spaces and $\pi: E \rightarrow B$ a map.

As usual, by a homotopy for the map $f: X \rightarrow Y$, we mean a map $F: X \times[0,1] \rightarrow$ $Y$ such that $F(x, 0)=f(x)$ for every $x \in X$; and we call $(E, \pi)$, or $\pi$, a fibration, or equivalently we say that it has the homotopy lifting property, if for every map $f: X \rightarrow E$ whose source $X$ is a polytope, every homotopy for $\pi \circ f$ lifts to a homotopy for $f$. More generally, call $\pi$ an $r$-fibration if this is true for every polytope $X$ of dimension at most $r$. A 0 -fibration is also said to have the path lifting property.

Here is another generalization of fibrations. Two homotopies

$$
F, F^{\prime}: X \times[0,1] \rightarrow Y
$$

are said to have the same germ if they coincide in a neighborhood of the subspace $X \times\{0\}$.

Received by the editors September 1, 2001 and, in revised form, October 20, 2001.

2000 Mathematics Subject Classification. Primary 55R05, 55R10.

(C)2002 American Mathematical Society 
Definition 1. Call $\pi$ a homotopic submersion, or equivalently say that it has the germ-of-homotopy lifting property, if for every map $f: X \rightarrow E$ whose source $X$ is a polytope, every germ-of-homotopy for $\pi \circ f$ lifts to a germ-of-homotopy for $f$.

Every fibration is a homotopic submersion (obvious), and so is every smooth submersion in the sense of differential geometry, and more generally every topological submersion (after lemma 6 below).

A "fibre" is the inverse image in $E$ of a point $b \in B$, denoted $E_{b}=\pi^{-1}(b)$. A map $X \rightarrow E$ is vertical if it sends $X$ into a single fibre. A map $f: X \times Y \rightarrow E$ is fibred if for each point $y$ in $Y$, the map

$$
f_{y}: X \rightarrow E: x \mapsto f(x, y)
$$

is vertical.

Definition 2. A vanishing p-cycle is a fibred map

$$
f: \mathbf{S}^{p} \times[0,1] \rightarrow E
$$

such that, for each $t>0$, the map $f_{t}$ is null-homotopic in its fibre. Call $f$ trivial if $f_{0}$ is also null-homotopic in its fibre.

Observe that $f$ has to be continuous, but that we don't demand that the homotopies, the vertical maps $g_{t}: \mathbf{B}^{p+1} \rightarrow E$ such that $\partial g_{t}=f_{t}$, depend continuously on $t$. Dually,

Definition 3. An emerging p-cycle is a fibred map

$$
\left.\left.f: \mathbf{S}^{p} \times\right] 0,1\right] \rightarrow E
$$

such that $f(*, t)$ has a limit for $t \rightarrow 0$ (recall that $*$ denotes the basepoint in $\mathbf{S}^{p}$ ). Call it trivial if there exist $\epsilon>0$ and a fibred map

$$
f^{\prime}: \mathbf{S}^{p} \times[0, \epsilon[\rightarrow E
$$

such that for each $0<t<\epsilon$ one has $f^{\prime}(*, t)=f(*, t)$, and such that the maps $f_{t}$ and $f_{t}^{\prime}$ are homotopic to each other in their common fibre, relatively to the basepoint $f(*, t)$.

Here also, we don't ask any continuity, relative to $t$, of the homotopy linking $f_{t}$ to $f_{t}^{\prime}$.

Thus a nontrivial vanishing (resp. emerging) cycle is a lack of injectivity (resp. surjectivity) in the homotopy groups of fibres, when moving from a given fibre to immediately neighbouring ones over some path in the base.

Write $V(X)$ for the space of vertical maps from $X$ into $E$, with the compact-open topology. Thus there is a canonical map

$$
\pi_{X}: V(X) \rightarrow B .
$$

Obviously, every $p$-dimensional vanishing (resp. emerging) cycle for the map $\pi$ can also be considered as a 0-dimensionnal vanishing (resp. emerging) cycle for the map $\pi_{\mathbf{S}^{p}}$. They are simultaneously trivial.

To end with precisions, the pullback of $\pi$ by some map $f: B^{\prime} \rightarrow B$ is, as usual, defined as $f^{*} \pi: f^{*} E \rightarrow B^{\prime}$, where $f^{*} E$ is the set of pairs $\left(e, b^{\prime}\right) \in E \times B^{\prime}$ such that $\pi(e)=f\left(b^{\prime}\right)$, and where $\left(f^{*} \pi\right)\left(e, b^{\prime}\right)=b^{\prime}$.

It is immediate that if $\pi$ is a fibration, or an $r$-fibration, or a homotopic submersion, or has only trivial $p$-vanishing, or emerging, cycles, then every pullback enjoys the same property. 
Example 4. The three conditions in theorem A are independent. Indeed, let $E \subset$ $[0,1]^{2}$ be defined by the condition $x y=0$ (resp. $x \neq 1 / 2$ or $\left.y \neq 1 / 2\right)($ resp. $y=0$ or $(y=1$ and $x \neq 0)$ ). Let $B=[0,1]$, and let $\pi$ be simply $(x, y) \mapsto x$. Then the first (resp. second) (resp. third) condition is not satisfied, but the other two are.

\section{I.2. Proof of theorem A.}

I.2.1. Three general lemmas about homotopic submersions. An immediate and wellknown fact about fibrations is that they automatically also have the relative homotopy lifting property. The same argument proves:

Lemma 5. Every homotopic submersion satisfies the relative germ-of-homotopy lifting property : for every polytope $X$, every subpolytope $X^{\prime} \subset X$, every map $f: X \rightarrow E$, every germ-of-homotopy $F$ for $\pi \circ f$ and every germ-of-homotopy $\bar{F}^{\prime}$ for $\left.f\right|_{X^{\prime}}$, if $\pi \circ \bar{F}^{\prime}=\left.F\right|_{X^{\prime}}$, then $\bar{F}^{\prime}$ extends to a germ-of-homotopy $\bar{F}$ for $f$ such that $\pi \circ \bar{F}=F$.

Lemma 6. Assume that $\pi$ is open and is a local homotopic submersion, i.e. that each $x \in E$ has a neighborhood $U(x) \subset E$ such that the restriction $\left.\pi\right|_{U(x)}$ is a homotopic submersion of $U(x)$ onto $\pi(U(x))$. Then $\pi$ is a (global) homotopic submersion.

Proof. Consider a polytope $X$, a map $f: X \rightarrow E$ and a germ-of-homotopy $F$ : $X \times[0, \epsilon[\rightarrow B$ for $\pi \circ f$. Proceed by induction on $p=\operatorname{dim} X$. The case $p=0$ is trivial. Subdividing $X$, we can assume that for each $p$-simplex $\sigma$, its image $f(\sigma)$ is contained in an open subset $U(\sigma) \subset E$ such that $\left.\pi\right|_{U(\sigma)}$ satisfies the germ-ofhomotopy lifting property. By induction, we have a lifting $\bar{F}^{\prime}$ for $F$ restricted to the $(p-1)$-skeleton of $X$. Since by the previous lemma $\left.\pi\right|_{U(\sigma)}$ has the relative germ-of-homotopy lifting property, $\bar{F}^{\prime}$ extends to the interior of $\sigma$.

A second immediate corollary of lemma 5 is the following tool for handling sections.

Lemma 7. Let $\pi: E \rightarrow B$ be a surjective homotopic submersion whose base $B$ is a polytope. Let $Y \subset B$ be a subpolytope. Denote by $\Gamma(Y)$ the space of (continuous) sections of $\pi$ over $Y$. Then:

(i: local section extension) Each $s \in \Gamma(Y)$ extends over some neighborhood of $Y$. In particular, each point of $B$ has a neighborhood over which there exists a section.

(ii: sections pasting) Let $s \in \Gamma(B)$ and let $s^{\prime} \in \Gamma(Y)$ be homotopic to $\left.s\right|_{Y}$. Then there exists $s^{\prime \prime} \in \Gamma(B)$ which coincides with s over the complement of an arbitrary small neighborhood of $Y$, and with $s^{\prime}$ on $Y$.

I.2.2. Haefliger's lemma. The following fact, due to Haefliger [4, is central in the demonstration of theorem A. We give a proof that seems simpler than the original one.

Lemma 8. If a surjective homotopic submersion $\pi: E \rightarrow B$ has a polytope base $B$ and weakly contractible fibres $E_{b}$, then it has a section.

Proof. By induction on $q=\operatorname{dim} B$. Obvious if $q=0$. Assume that the lemma is true for $q-1$. Since a surjective homotopic submersion has local sections, subdividing $B$ if necessary, every $q$-cell $\sigma$ is the domain of a section $s_{\sigma}$. On the other hand, the induction hypothesis gives a section $s$ over the $(q-1)$-skeleton. Over the boundary of each $q$-cell $\sigma$, the two sections $\left.s\right|_{\partial \sigma}$ and $\left.s_{\sigma}\right|_{\partial \sigma}$ are homotopic. 
Indeed, consider the space $E^{\prime}$ of vertical paths $c:[0,1] \rightarrow E_{b}$ such that $b \in \partial \sigma$, $c(0)=s_{\sigma}(b)$ and $c(1)=s(b)$. Obviously the map $c \mapsto b$ is a homotopic submersion of $E^{\prime}$ onto $\partial \sigma$ with weakly contractible fibres, so by the induction hypothesis it admits a section, i.e. a homotopy between $\left.s\right|_{\partial \sigma}$ and $\left.s_{\sigma}\right|_{\partial \sigma}$. Thus the section pasting property of the homotopic submersion $\pi$ gives an extension of $s$ over $\sigma$.

I.2.3. About the sections of a homotopic submersion over the interval. In this subsection, consider a surjective homotopic submersion whose base is the interval:

$$
\pi: E \rightarrow B=[0,1] .
$$

Fix a basepoint $x_{0} \in E_{0}$. For short, call a section pointed if $s(0)=x_{0} ;$ write $\Gamma(Y)$ (resp. $\Gamma_{0}(Y)$ ) for the space of (pointed) sections over $Y \subset I$; and call $s \in \Gamma(Y)$, $s^{\prime} \in \Gamma\left(Y^{\prime}\right)$ compatible at a point $b \in Y \cap Y^{\prime}$ if $s(b)$ and $s^{\prime}(b)$ belong to the same arcwise connected component of $E_{b}$.

Lemma 9. Assume that all 0-vanishing cycles are trivial. If $s, s^{\prime} \in \Gamma([0,1])$ are compatible at some point, then they are compatible at every point.

Proof. Recall that if $K \subset[0,1]$ is a compact subset, we call $t \in \mathbf{K}$ a first-species point of $K$ if $t$ is an extremity of a nonempty open interval disjoint from $K$.

The set $U \subset[0,1]$ of points where $s$ and $s^{\prime}$ are compatible is open since $\pi$ is a homotopic submersion, and its complement has no first-species point since 0 vanishing cycles are trivial. Thus $U=[0,1]$.

Lemma 10. Assume that all 0-vanishing cycles and 0-emerging cycles are trivial. Then $\Gamma_{0}([0,1])$ is not empty.

Proof. Consider the set $\mathbf{T}$ of $t$ 's such that there exists a pointed section over $[0, t]$. It is open since $\pi$ is a homotopic submersion. Thus by contradiction it would be of the form $\mathbf{T}=[0, T[$ with $T \in] 0,1]$. Let $s_{n} \in \Gamma([0, T-1 / n])$ be pointed. By the previous lemma, since $s_{n}(0)=s_{n+1}(0)$, the sections $s_{n}$ and $s_{n+1}$ are also compatible at the point $T-1 / n$. Thus the sections pasting property gives a pointed $s \in \Gamma\left(\left[0, T[)\right.\right.$. Let $\left.\left.s^{\prime} \in \Gamma(] T-\epsilon, T\right]\right)$ be any section over a left neighborhood of $T$. Then $s$ and $s^{\prime}$ together form a 0 -emerging cycle at $T$. Since it must be trivial, there is an $s^{\prime \prime} \in \Gamma\left(\left[T-\epsilon^{\prime}, T\right]\right)$ compatible with $s$ at $t-\epsilon^{\prime}$. The sections pasting property gives a pointed section over $[0, T]$, contradiction.

Lemma 11. Any surjective homotopic submersion (with arbitrary base) all of whose vanishing 0-cycles and emerging 0-cycles are trivial, is a 0-fibration.

Proof. Immediate by lemma 10 and pullbacks.

Lemma 12. Assume that all vanishing cycles and emerging cycles of all dimensions are trivial. Then $\Gamma_{0}([0,1])$ is weakly contractible.

Proof. For every polytope $X$, recall $V(X)$, the space of vertical maps $X \rightarrow E$. The canonical map $\pi_{X}: V(X) \rightarrow[0,1]$ is a homotopic submersion, and so is the restriction map

$$
\rho^{p}: V\left(\mathbf{B}^{p+1}\right) \rightarrow V\left(\mathbf{S}^{p}\right) .
$$

Since we assumed that all vanishing (resp. emerging) cycles of $\pi$ of dimension $p+1$ are trivial, it follows that all 0-dimensionnal vanishing (resp. emerging) cycles of $\rho^{p}$ are also trivial. 
Indeed, let $f: \mathbf{S}^{0} \times[0,1] \rightarrow V\left(\mathbf{B}^{p+1}\right)\left(\right.$ resp. $\left.\left.\left.f: \mathbf{S}^{0} \times\right] 0,1\right] \rightarrow V\left(\mathbf{B}^{p+1}\right)\right)$ be a vanishing (resp. emerging) 0 -cycle for $\rho^{p}$. Gluing, for each $t \in[0,1]$ (resp. each $t \in] 0,1]), f(-1, t)$ and $f(+1, t)$ along their common boundary, one gets a vanishing (resp. emerging) $(q+1)$-cycle for $\pi$, the triviality of which implies that $f$ is also trivial.

Thus by lemma $11, \rho^{p}$ is a 0 -fibration as a map $V\left(\mathbf{B}^{p+1}\right) \rightarrow V^{0}\left(\mathbf{S}^{p}\right)$, where $V^{0}\left(\mathbf{S}^{p}\right)$ is the image of $\rho^{p}$, i.e. the space of vertical $p$-cycles null-homotopic in their fibre.

Every given map $g: \mathbf{S}^{p} \rightarrow \Gamma_{0}([0,1])$ may also be considered as a section $s$ : $[0,1] \rightarrow V\left(\mathbf{S}^{p}\right)$ (set $\left.s(t)(x)=g(x)(t)\right)$. The set of $t$ 's such that $s(t) \in V^{0}\left(\mathbf{S}^{p}\right)$ is open since $\pi$ is a homotopic submersion, and has no first-species boundary point since $p$-vanishing cycles are trivial. Thus $s$ is a path in $V^{0}\left(\mathbf{S}^{p}\right)$, and thus lifts to $V\left(\mathbf{B}^{p+1}\right)$, i.e. $g$ extends to $\mathbf{B}^{p+1}$.

I.2.4. End of the proof of theorem $A$. Given a map $\pi: E \rightarrow \mathbf{B}$ satisfying the three conditions of the theorem, a map $f: X \rightarrow E$ and a homotopy $F$ for $\pi \circ f$, consider the space $E^{\prime}$ of pairs $(x, c)$ where $x \in X$ and where $c:[0,1] \rightarrow E$ satisfies $c(0)=f(x)$ and $\pi(c(t))=F(x, t)$ for every $t \in[0,1]$. Then

$$
\pi^{\prime}: E^{\prime} \rightarrow X:(x, c) \mapsto x
$$

is a homotopic submersion, as follows from lemma 5 . It is surjective by lemma 10 , and its fibres are weakly contractible by lemma 12 . By lemma 8 , this map $\pi^{\prime}$ admits a section; in other words, $F$ lifts.

I.3. Corollaries. The two first immediately follow from theorem A.

Corollary 13. Let $\pi: E \rightarrow B$ be a surjective homotopic submersion. Under either of the following assumptions, $\pi$ is a fibration:

1. Each fibre is weakly contractible.

2. The inclusion of each fibre into $E$ is a weak homotopy equivalence.

Surprisingly, point 1, essentially identical to Haefliger's lemma, seems to be the only one of our various corollaries to have been previously known.

Corollary 14. Let $\pi: E \rightarrow B$ be a surjective homotopic submersion. The following properties are equivalent:

1. The map $\pi$ is a fibration.

2. For each $b$ in $B$, the map $\pi$ induces a weak homotopy equivalence of pairs $\left(E, E_{b}\right) \rightarrow(B, b)$.

3. (dim-1 base principle for fibrations) For each path $c:[0,1] \rightarrow B$, the pullback $c^{*} E$ is a fibration over $[0,1]$.

The dim-1 base principle allows us, whenever useful, to restrict our attention to the case $B=[0,1]$.

Corollary 15. In a commutative diagram of maps

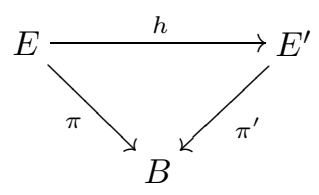

assume that $\pi$ and $\pi^{\prime}$ are surjective homotopic submersions. For each $b \in B$, consider $E_{b}=\pi^{-1}(b), E_{b}^{\prime}=\pi^{\prime-1}(b)$, and the restriction $h_{b}=\left.h\right|_{E_{b}}: E_{b} \rightarrow E_{b}^{\prime}$. 
(i) (Fibrewise fibration principle) If $\pi, \pi^{\prime}$ and every $h_{b}$ are fibrations and if $h$ is a surjective homotopic submersion, then $h$ is a fibration.

(ii) If $\pi$ is a fibration and if every $h_{b}$ is a weak homotopy equivalence, then $\pi^{\prime}$ is a fibration.

(iii) If $\pi^{\prime}$ is a fibration and if every $h_{b}$ is a weak homotopy equivalence, then $\pi$ is a fibration.

Proof. (i) We can assume, to simplify, that $B$ is contractible (for example using the dim-1 base principle). Fix $x^{\prime} \in E^{\prime}$, write $b=\pi^{\prime}\left(x^{\prime}\right), E_{x^{\prime}}=h^{-1}\left(x^{\prime}\right)$, and consider the inclusions $j: E_{b} \rightarrow E$ and $j^{\prime}: E_{b}^{\prime} \rightarrow E^{\prime}$. We have a commutative diagram of pairs :

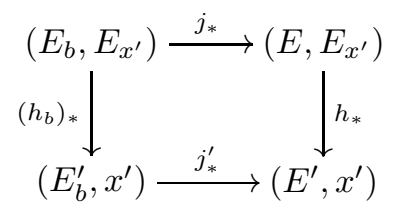

Since $\pi, \pi^{\prime}$ and $h_{b}$ are fibrations, $j_{*}, j_{*}^{\prime}$ and $\left(h_{b}\right)_{*}$ are weak homotopy equivalences, and thus so is $h_{*}$. Corollary 14 now yields the desired conclusion.

(ii) Using the dim-1 base principle, we can assume, to fix ideas, that $B=[0,1]$. Then we have a section-lifting lemma :

Lemma 16. For every subinterval $I \subset[0,1]$ and every section $s^{\prime}: I \rightarrow E^{\prime}$, there is a section $s: I \rightarrow E$ compatible with $s^{\prime}$, i.e. such that for every $t \in I$, the points $h(s(t))$ and $s^{\prime}(t)$ lie in the same arcwise connected component of $E_{t}^{\prime}$.

Proof of the lemma. Since every $h_{b}$ is a 0-homotopy equivalence, there exists a settheoretic (not necessarily continuous) section $\sigma: I \rightarrow E$ compatible with $s^{\prime}$. Since $\pi$ is a homotopic submersion, each $t \in I$ has a neighborhood $U(t)$ in $I$ with a (continuous) section $s_{t}: U(t) \rightarrow E$ such that $s_{t}(t)=\sigma(t)$. Since $\pi^{\prime}$ is a homotopic submersion, $s_{t}$ is compatible with $s^{\prime}$ over some smaller neighborhood of $t$. Thus $I$ can be divided into subintervals $I_{i}=\left[t_{i}, t_{i+1}\right]$, domains of sections $s_{i}: I_{i} \rightarrow E$ compatible with $s^{\prime}$. In particular, since $h_{t_{i}}$ is a 0 -homotopy equivalence, $s_{i-1}\left(t_{i}\right)$ and $s_{i}\left(t_{i}\right)$ lie in the same arcwise connected component of $E_{t_{i}}$. Thus the section pasting tool (lemma 7) allows us to build a section $s: I \rightarrow E$ compatible with $s^{\prime}$. The lemma is proved.

End of the proof of corollary 15. Since 0-vanishing and 0-emerging cycles of $\pi$ are trivial, and since every $h_{b}$ is a 0 -homotopy equivalence, the section-lifting lemma gives straightforwardly that 0 -vanishing and 0-emerging cycles of $\pi^{\prime}$ are trivial.

Changing $E$ for $V\left(\mathbf{S}^{p}\right)$, the space of vertical maps $\mathbf{S}^{p} \rightarrow E$, and changing $E^{\prime}$ for $V^{\prime}\left(\mathbf{S}^{p}\right)$, the space of vertical maps $\mathbf{S}^{p} \rightarrow E^{\prime}$, we get that $p$-vanishing and $p$-emerging cycles of $\pi^{\prime}$ are trivial, for every $p \geq 0$. Theorem A now yields the desired conclusion.

(iii) Much like (ii), but simpler.

Corollary 17. Let $\pi: E \rightarrow B$ be a surjective homotopic submersion. The map $\pi$ is a fibration if and only if the canonical map from each fibre into the homotopytheoretic fibre of $\pi$ is a weak homotopy equivalence.

This follows immediately from part (iii) of corollary 15. 
We can also deduce two characterizations for product maps. Of course, we call $\pi: E \rightarrow B$ a fibration over a given subset $B^{\prime} \subset B$ if the restriction

$$
\left.\pi\right|_{\pi^{-1}\left(B^{\prime}\right)}: \pi^{-1}\left(B^{\prime}\right) \rightarrow B^{\prime}
$$

is a fibration.

Corollary 18. Assume that $\pi=\left(\pi_{1}, \pi_{2}\right): E \rightarrow B=B_{1} \times B_{2}$ is a surjective homotopic submersion. The three following properties are equivalent:

(i) The map $\pi$ is a fibration.

(ii) The map $\pi$ is, for each $b_{1} \in B_{1}$, a fibration over $\left\{b_{1}\right\} \times B_{2}$; and $\pi_{1}$ is a fibration.

(iii) The map $\pi$ is, for each $b_{1} \in B_{1}$, a fibration over $\left\{b_{1}\right\} \times B_{2}$; and, for each $b_{2} \in B_{2}$, a fibration over $B_{1} \times\left\{b_{2}\right\}$.

Proof. The equivalence of (i) and (ii) is a special case of the fibrewise fibration principle (corollary 15). It is obvious that (i) implies (iii).

Let us assume that (iii) is true. To prove (i), we can, using the dim-1 base principle, assume that $B_{2}=[0,1]$. For every $b_{1} \in B_{1}$, since $\pi$ is a fibration over $\left\{b_{1}\right\} \times B_{2}$ which is contractible, it follows that $\pi^{-1}\left(b_{1}, 0\right)$ is a deformation retract of $\pi^{-1}\left(\left\{b_{1}\right\} \times B_{2}\right)=\pi_{1}^{-1}\left(b_{1}\right)$.

On the other hand, since $\pi$ is a fibration over $\pi_{2}^{-1}(0)$, equivalently $\pi_{1}$ restricted to $\pi_{2}{ }^{-1}(0)$ is a fibration.

It follows from corollary 15 (ii) that $\pi_{1}$ is also a fibration. Thus (ii) is verified. Thus (i) is also.

The next and last corollary, two refined forms of the dim-1 base principle, will be a crucial tool in part II.

Corollary 19. Let $\pi: E \rightarrow B$ be a surjective homotopic submersion.

a) Assume that $B=\mathbf{R}^{q}$. Then $\pi$ is a fibration if and only if it is a fibration over every straight line in the base parallel to one of the axes.

b) Assume that $B$ is a $C^{\infty}$ (resp. real analytic) (resp. PL) manifold. Then $\pi$ is a fibration if and only if its pullback by every $C^{\infty}$ (resp. real analytic) (resp. PL) path in $B$ is a fibration over $[0,1]$.

Proof. a) is immediate by the preceding corollary and an induction on $q$ and b) follows immediately from a).

Example 20. We exhibit a case in which $\pi=\left(\pi_{1}, \pi_{2}\right)$ is not a fibration (its fibres don't have the same homotopy type) although it is a surjective submersion and $\pi_{1}$, $\pi_{2}$ are both fibrations (their fibres are contractible) :

$$
E=\left\{(x, y, z) \in \mathbf{R}^{3} \mid z \neq 0 \text { or } x>y\right\}
$$

and $\pi: E \rightarrow B=\mathbf{R}^{2}:(x, y, z) \mapsto(x, y)$.

\section{Submersions And Bundles}

In this second part we turn to submersions between manifolds, establish a necessary and sufficient condition for such a map to be a (locally trivial fibre) bundle (theorem B below), and apply it to several typical situations. The main tool is the analytic understanding of fibrations we got in $\S \mathrm{I}$. It is applied to spaces of embeddings of compact domains into the fibres. 
The first question that arises is probably: For such a submersion, is being a fibration the same as being a bundle? It was considered by Ferry [3], in a framework slightly different from ours; he built counterexamples. On the other hand, Haefliger's lemma allows much simpler ones, e.g.:

Example 21. Let $W \subset \mathbf{R}^{3}$ be the Whitehead manifold - thus an open subset which is contractible, but has some $\pi_{1}$ at infinity, and in particular is not homeomorphic to $\mathbf{R}^{3}$. Let $E \subset \mathbf{R}^{4}$ be the set of quadruples $(x, y, z, t)$ such that $(x, y, z) \in W$ or $t \neq 0$. Let

$$
\pi: E \rightarrow B=\mathbf{R}:(x, y, z, t) \mapsto t .
$$

Then $p$ is a smooth submersion (since $E$ is open in $\mathbf{R}^{4}$ ) and a fibration (since all fibres are contractible, see $\S \mathrm{I}$ ). But it is certainly not a locally trivial fibre bundle, since one of the fibres is not homeomorphic to the neighbouring ones.

II.1. Definitions. To fix ideas, work in the smooth $\left(C^{\infty}\right.$ real $)$ differentiability class. Let $E^{p+q}, B^{q}$ be manifolds - this means paracompact, not necessarily compact, real differential manifolds. For simplicity, assume that $E, B$ are without boundary. Let $\pi: E \rightarrow B$ be a surjective smooth submersion - that is, its differential at every point of $E$ is onto. Then each "fibre" $E_{b}=\pi^{-1}(b)$ is a $p$ manifold.

Call a submanifold of $E$ a product if it is the image of a fibred (see $\S \mathrm{I}$ ) smooth embedding $X \times Y \rightarrow E$. Recall that $\pi$ is a (locally trivial, fibre) bundle if every $b \in B$ has a neighborhood $Y$ such that $\pi^{-1}(Y)$ is a product.

A popular sufficient condition for $\pi$ to be a bundle is that $\pi$ be a riemannian submersion [5], [11]. Recall that a riemannian metric on $E$ is called bundle-like if there exists a riemannian metric on the base $B$ such that, for each $b \in B$ and each $x \in E_{b}$, the differential $D_{x} \pi$ establishes a linear isometry from the normal vector space $\nu_{x} E_{b}$ onto the tangent vector space $T_{b} B$. It is easy, using a partition of the unity, to make a (maybe noncomplete) bundle-like metric. The 'orthogonal geodesic lemma' asserts that if any geodesic line is once normal to the fibre it crosses, then it is forever. It follows easily that if $X \subset E_{b}$ is an open subset (to fix ideas) such that the exponential $\exp \left(v_{x}\right)$ is defined for all $x \in X$ and all $v_{x} \in \nu_{x} E_{b}$ with norm less than a uniform positive constant, then the set of all these $\exp \left(v_{x}\right)$ 's is a product. In particular we get the fundamental

Lemma 22. Every compact subset of every fibre has a product neighborhood.

One calls $\pi$ riemannian if $E$ admits a complete bundle-like metric, and, taking $X=E_{b}$ above, one sees that every riemannian submersion is a bundle. There are many generalizations.

Our viewpoint is different: we look for purely differential-topological conditions in terms of the topology of the fibres. In this direction, very little seems to be known, namely:

1. If $\pi$ is proper, then it is a bundle (Ehresmann). This follows at once from lemma 22.

2. If all the leaves are compact and have the same number of connected components, then $\pi$ is a bundle. This also is obvious by lemma 22 .

3. If each fibre is diffeomorphic to $\mathbf{R}$, then $\pi$ is a bundle. Indeed, one may assume that $B$ is orientable. Using a partition of unity, make a nonsingular vector field tangent to the fibres, make it integrable, and integrate it. 
4. Much less elementary is Palmeira's lemma 9]: if each fibre is diffeomorphic to $\mathbf{R}^{p}, p$ a nonnegative integer, and if the base $B$ has dimension $q=1$, then $\pi$ is a bundle.

A fibred embedding of $X \times Y$ into $E$ can be considered as a section, over some subset of $B$, with values in the space $V E(X, E)$ of vertical embeddings of $X$ into $E$. Thus an approach could be to start from a large compact domain $X$ in a fibre and from a ball $Y \subset B$, to use our knowledge of the topology of the fibres to compute as much as possible the homotopy type of the space of embeddings of $X$ into each fibre, and to use $\S I$ to get such sections. Of course there would remain the problem to engulf arbitrary large compact subsets of $\pi^{-1}(Y)$. For instance, in example 21, the canonical map $V E\left(\mathbf{B}^{3}, E\right) \rightarrow B$ is a fibration (see the proof of corollary 31 below) and nevertheless the vertical embeddings of $\mathbf{B}^{3}$ exhaust all fibres but $E_{0}$. This leads us to the following notions.

By a vertical domain we mean a $p$-dimensionnal compact submanifold of a fibre, $X \subset E_{b}$, with a smooth boundary. Endow $V E(X, E)$, the set of vertical embeddings of $X$ into $E$, with the topology of smooth uniform convergence. Let $V E^{0}(X, E)$ be the connected component of $V E(X, E)$ containing the original inclusion $X \rightarrow E_{b}$.

Definitions 23. Let

$$
\mathrm{VD}=\coprod_{b \in B} \mathrm{VD}_{b}
$$

be a family of vertical domains. Call it:

a) exhaustive if every compact subset of every fibre is contained in some $X \in \mathrm{VD}$;

b) isotopy invariant if for every $X \in \mathrm{VD}$ and every $\phi \in V E^{0}(X, E)$ we have $\phi(X) \in \mathrm{VD}$

c) $r$-fibred if, for any two domains $X, X^{\prime} \in \mathrm{VD}_{b}$ such that $X \subset \operatorname{Int}\left(X^{\prime}\right)$, the restriction map

$$
\rho_{X, X^{\prime}}: V E^{0}\left(X^{\prime}, E\right) \rightarrow V E^{0}(X, E)
$$

is an $r$-fibration (i.e., has the homotopy lifting property for polytopes of dimension at most $r$, see $\S \mathrm{I})$.

\section{II.2. Characterizations and criteria for bundles.}

Theorem B. A surjective smooth submersion $\pi: E \rightarrow B^{q}$ is a (locally trivial fibre) bundle if and only if it admits an exhaustive, isotopy invariant, $(q-1)$-fibred family of vertical domains.

Before the proof, let us give some definitions and preliminary lemmas.

Lemma 24. For every pair of domains $X \subset \operatorname{Int}\left(X^{\prime}\right)$, the restriction map $\rho_{X, X^{\prime}}$ is a topological submersion.

This follows at once from lemma 22 .

For every vertical domain $X \in \mathrm{VD}_{b}$ and every subset $Y \subset B$ homeomorphic to $\mathbf{B}^{k}$ for some $0 \leq k \leq q$, write $\Gamma(X, Y)$ for the space of continuous sections $\gamma: Y \rightarrow V E^{0}(X, E)$. Observe in particular that for $\gamma \in \Gamma(X, Y)$, the variable point $\gamma(x)(y)$ admits continuous partial derivates of all orders with respect to $x$, bur not necessarily with respect to $y$. Since we shall perform nonstrictly-differentiable 
operations on the base, it is more convenient to consider all those sections than merely smooth ones. Write

$$
\operatorname{Im} \gamma=\{\gamma(y)(x) \mid x \in X, y \in Y\} .
$$

Call $\gamma$ a parametrization of the VD-box $\operatorname{Im} \gamma$.

Recall that $X \subset E_{b}$. Call $\gamma$ pointed (at the point $b$ ) if $b \in Y$ and $\gamma(b)=\operatorname{id}_{X}$.

For every compact subset $C \subset E$, write $C<\operatorname{Im} \gamma$ if $C \subset \operatorname{Im} \gamma$ and $C \cap E_{y} \subset$ $\operatorname{Int}\left(\operatorname{Im} \gamma \cap E_{y}\right)$ for every $y \in Y$.

Given two sections $\gamma \in \Gamma(X, Y), \gamma^{\prime} \in \Gamma\left(X^{\prime}, Y^{\prime}\right)$, we say of course that $\gamma^{\prime}$ extends $\gamma$ if $X \subset X^{\prime}, Y \subset Y^{\prime}$, and $\gamma(y)(x)=\gamma^{\prime}(y)(x)$ for every $x \in X, y \in Y$.

Lemma 25. If $\gamma$ is pointed and if $\operatorname{Im} \gamma<\operatorname{Im} \gamma^{\prime}$, then there exists a pointed reparametrization of $\operatorname{Im} \gamma^{\prime}$ extending $\gamma$.

Since the bases are contractible, this is an exercise using the classical [1]

Proposition 26. Let $X$ and $X^{\prime}$ be compact manifolds with $X \subset \operatorname{Int}\left(X^{\prime}\right)$. Let Dif $f^{0}\left(X^{\prime}\right)$ denote the group of isotopies of $X^{\prime}$, and $E^{0}\left(X, X^{\prime}\right)$ the canonical connected component of the space of embeddings of $X \rightarrow X^{\prime}$. Then the restriction map Dif $f^{0}\left(X^{\prime}\right) \rightarrow E^{0}\left(X, X^{\prime}\right)$ is a fibration (and even a principal bundle).

Here is the main tool to engulf large compact subsets.

Lemma 27. Let $K_{i} \subset E(i=1,2,3)$ be three VD-boxes with base $Y_{i}=\pi\left(K_{i}\right)$. Let $r=\max \left(\operatorname{dim} Y_{1}, \operatorname{dim} Y_{2}\right)-1$. Assume that $Y_{1} \cap Y_{2}=Y_{3}$, that $K_{i} \cap \pi^{-1}\left(Y_{3}\right)<K_{3}$ for $i=1,2$, and that $\mathrm{VD}$ is $r$-fibred. Then there is a VD-box $K_{4}$ such that $K_{i}<K_{4}$ for $i=1,2$.

Proof. Fix a basepoint $b \in Y_{3}$. For $i=1,2,3$, let $X_{i}=K_{i} \cap E_{b}$ and let $\gamma_{i} \in$ $\Gamma\left(X_{i}, Y_{i}\right)$ be a pointed parametrization of $K_{i}$. According to lemma 25 , we can assume that $\gamma_{3}$ extends $\left.\gamma_{1}\right|_{Y_{3}}$.

Since VD is $r$-fibred and $Y_{1}, Y_{3}$ are balls of dimension $\leq r-1$, there exists a pointed $\gamma_{1}^{\prime} \in \Gamma\left(X_{3}, Y_{1}\right)$, extending $\gamma_{1}$, and which coincides with $\gamma_{3}$ over $Y_{3}$. According to lemma 25, there is a reparametrization $\gamma_{1}^{\prime \prime}$ of $\gamma_{1}^{\prime}$ such that $\left.\gamma_{1}^{\prime \prime}\right|_{Y_{3}}$ extends $\left.\gamma_{2}\right|_{Y_{3}}$.

In the same way, there exists a $\gamma_{2}^{\prime} \in \Gamma\left(X_{3}, Y_{2}\right)$ extending $\gamma_{2}$ and which coincides with $\gamma_{1}^{\prime \prime}$ over $Y_{3}$.

Since $\gamma_{1}^{\prime \prime}$ and $\gamma_{2}^{\prime}$ coincide over $Y_{3}$, they define an element of $\Gamma\left(X_{3}, Y_{1} \cup Y_{2}\right)$, whose image $K_{4}$ is obviously $>K_{1}$ and $>K_{2}$.

Proof of theorem B. "Only if" is trivial: just take for VD the set of all vertical domains. Thus, reciprocally, we assume that $\pi: E \rightarrow B$ is a smooth surjective submersion which admits an exhaustive, isotopy-invariant, $(q-1)$-fibred set VD of vertical domains, and let us prove that it is a fibration. For every $b \in B$, let $Y$ be a neighborhood of $b$ diffeomorphic to $\mathbf{B}^{q}$. We first claim:

Every compact subset $C \subset \pi^{-1}(Y)$ is $<$ some VD-box.

The proof is by induction on $k$, the smallest integer such that there exists $Y^{k}$ homeomorphic to $\mathbf{B}^{k}$ and satisfying $\pi(C) \subset Y^{k} \subset Y$.

For $k=0$ the claim follows from the exhaustiveness of VD.

Assume that the claim is proved for $k-1$. Identify $Y^{k}$ with $[0,1]^{k}$. For each $y \in Y$, by exhaustiveness of VD there is a vertical domain belonging to VD and containing $C \cap E_{y}$ in its interior. By lemma 24 this domain is the intersection of $E_{y}$ with a VD-box $K_{y}$ whose base $\pi\left(K_{y}\right)$ is a neighborhood of $y$. Since $C$ is compact, 
$y$ admits a smaller neighborhood $W_{y}$ such that $C \cap \pi^{-1}\left(W_{y}\right)<K_{y}$. Thus dividing $Y^{k}=[0,1]^{k}$ into small enough cubes of equal size $1 / N$, say $Q_{1}, \ldots, Q_{N^{k}}$, each $C \cap \pi^{-1}\left(Q_{i}\right)$ is $<$ some VD-box, say $K_{i}$, of base $\pi\left(K_{i}\right)=Q_{i}$.

Consider $U_{i}=Q_{1} \cup \cdots \cup Q_{i}$ the union of the first $i$ small cubes (ranged of course in the natural lexicographic order). Assume by induction on $i$ that $C \cap \pi^{-1}\left(U_{i}\right)$ is $<$ some VD-box $K_{i}^{\prime}$ of base $\pi\left(K_{i}^{\prime}\right)=U_{i}$. The intersection $U_{i} \cap Q_{i+1}$ is obviously homeomorphic to $\mathbf{B}^{k-1}$; thus, by the induction (on $k$ ) hypothesis, $\left(K_{i} \cup K_{i}^{\prime}\right) \cap \pi^{-1}\left(U_{i} \cap Q_{i+1}\right)$ is $<$ a VD-box. Since moreover $U_{i}$ and $Q_{i+1}$ are contractible and since VD is $(k-1)$-fibred, lemma 27 assures the existence of a VD-box $K_{i+1}^{\prime}>C \cap \pi^{-1}\left(U_{i+1}\right)$. For $i=N^{k}-1$, we are done : the claim is proved.

Fix an increasing sequence $\left(C_{n}\right)$ of compact sets whose union is $\pi^{-1}(Y)$. The previous claim gives a sequence of pointed sections $\xi_{n} \in \Gamma\left(X_{n}, Y\right)$, with $X_{n} \in \mathrm{VD}_{b}$, such that

$$
C_{n} \cup \operatorname{Im} \xi_{n-1}<\operatorname{Im} \xi_{n}
$$

Changing if necessary $\xi_{n}$ to another section close to it, we can assume that the embedding $(x, y) \mapsto \xi_{n}(y)(x)$ is smooth. By lemma 25, a convenient reparametrization of $\xi_{n}$ gives $\xi_{n}$ as an extension of $\xi_{n-1}$. Consider $\xi$, the inductive limit of the $\xi_{n}$ 's . Its image is $\pi^{-1}(Y)$, since it contains every $C_{n}$. In other words, $\xi$ is a smooth trivialization of $E$ over $Y$.

Of course, in practice it may be hard to verify that a map such as $\rho_{X, X^{\prime}}$ is $(q-1)$ fibred, since, after part I, this is something like comparing the $(q-1)$-homotopy type of the embedding spaces of domains into the different fibres. So our next tasks will be to change this condition to more handy ones.

Corollary 28 (dim-1 base principle for bundles). a) A surjective smooth submersion $\pi: E \rightarrow B=\mathbf{R}^{q}$ is a bundle iff it is a bundle over each straight line in the base parallel to one of the axes.

b) A surjective smooth submersion $\pi: E \rightarrow B$ is a bundle iff for every smooth path $\gamma:[0,1] \rightarrow B$ the pullback $\gamma^{*} \pi: \gamma^{*} E \rightarrow[0,1]$ is a bundle.

c) Theorem $B$ is still true if we change " $(q-1)$-fibred" to "0-fibred".

Proof. To prove a), we make an induction on $q$. Assume that $\pi: E \rightarrow \mathbf{R}^{q}$ is a surjective submersion and a bundle over each straight line $L$ parallel to an axis. Let VD be the set of all vertical domains. By theorem B, it is enough to prove that $\rho: V E^{0}\left(X^{\prime}, E\right) \rightarrow V E^{0}(X, E)$ is a fibration for any two vertical domains $X$, $X^{\prime} \in \mathrm{VD}$ such that the first one is contained in the interior of the second one.

By assumption, for every $L$, our submersion $\pi$ is a bundle over $L$, and in particular the map $V E^{0}\left(X, \pi^{-1}(L)\right) \rightarrow L$ is a fibration. By corollary 19,

$$
\pi_{X}: V E^{0}(X, E) \rightarrow \mathbf{R}^{q}
$$

is a fibration. Write $\pi_{X}=\left(\pi_{X}^{1}, \ldots, \pi_{X}^{q}\right)$. Thus the last factor,

$$
\pi_{X}^{q}: V E^{0}(X, E) \rightarrow \mathbf{R}
$$

is also a fibration.

In the same way, $\pi_{X^{\prime}}^{q}$ is also a fibration.

But by the induction hypothesis, $\rho$ is a fibration over $\left(\pi_{X^{\prime}}^{q}\right)^{-1}(t)$ for each $t \in \mathbf{R}$. The fibrewise fibration principle (corollary 15 , (i)) says that $\rho$ is actually a fibration.

Affirmation b) then follows immediately from a); and affirmation c) from theorem $\mathrm{B}$ and from $\mathrm{b})$. 
Also, since our theorem A gave a satisfying analysis of fibrations, one can assume that $\pi$ is a submersion and a fibration, say a "submersion-fibration," and ask for sufficient conditions which make it a bundle.

Definition 29. Call VD engulfing when for every trio of domains $X, X^{\prime}, X^{\prime \prime} \in$ $\mathrm{VD}_{b}$ such that $X \subset \operatorname{Int}\left(X^{\prime}\right) \cap \operatorname{Int}\left(X^{\prime \prime}\right)$, if, in the ambiant space $E_{b}$, the domain $X^{\prime}$ can be pushed into $X^{\prime \prime}$ by a homotopy relative to $X$, then it can also be pushed into $X^{\prime \prime}$ by an isotopy relative to $X$.

Observe that we only ask the homotopy, and the isotopy, to be the identity on $X$, rather than on the whole of $X^{\prime} \cap X^{\prime \prime}$.

Corollary 30. Let $\pi: E \rightarrow B^{q}$ be a (surjective, smooth) submersion-fibration. If it admits an exhaustive, isotopy-invariant, engulfing set of vertical domains, then it is a bundle.

Proof. After the preceding corollary, we just have to prove that VD has the path lifting property. After a convenient pullback, $B=[0,1]$, we are given $X, X^{\prime} \in \mathrm{VD}_{0}$ such that $X \subset \operatorname{Int}\left(X^{\prime}\right) \subset X^{\prime}$ and $\xi \in \Gamma(X,[0,1])$ pointed, and we have to build a pointed $\xi^{\prime} \in \Gamma\left(X^{\prime},[0,1]\right)$ such that $\rho_{X, X^{\prime}}\left(\xi^{\prime}\right)=\xi$. Consider the set $\mathbf{T}$ of $t^{\prime}$ 's such that there exists a pointed $\xi_{t}^{\prime} \in \Gamma\left(X^{\prime},[0, t]\right)$ such that $\rho_{X, X^{\prime}}\left(\xi^{\prime}\right)=\left.\xi\right|_{[0, t]}$. Obviously $\mathbf{T}$ is an interval, open by lemma 24, and containing 0 . Assume, by contradiction, that $\mathbf{T}=[0, T[$. Since $\pi$ is a fibration, there is a pointed section $\gamma$ of the map $V^{0}\left(X^{\prime}, E\right) \rightarrow[0,1]$, where $V^{0}\left(X^{\prime}, E\right)$ is the space of vertical maps $X^{\prime} \rightarrow E$ homotopic to $\mathrm{id}_{X^{\prime}}$. Since $\mathrm{VD}$ is exhaustive, there is a $X^{\prime \prime} \in \mathrm{VD}_{T}$ containing $\gamma(T)\left(X^{\prime}\right)$ in its interior. By lemma 24, for $t<T$ close enough to $T$ there is a $\xi^{\prime \prime} \in \Gamma\left(X^{\prime \prime},[t, T]\right)$ such that $\xi^{\prime \prime}(T)=\operatorname{id}_{X^{\prime \prime}}$.

In the fibre $E_{t}$, we have three domains $\xi(t)(X), \xi^{\prime}(t)\left(X^{\prime}\right), \xi^{\prime \prime}(t)\left(X^{\prime \prime}\right)$; and since $\pi$ is a fibration, the second one can be pushed into the third one by a homotopy relative to the first one. Since VD is engulfing, the second one can also be pushed into the third one by an isotopy relative to the first one. In other words $\xi^{\prime}(t)$ is isotopic in $E_{t}$, relative to $\xi(t)(X)$, to some embedding $\phi: X^{\prime} \rightarrow \xi^{\prime \prime}(t)\left(X^{\prime \prime}\right)$. The section

$$
s \mapsto \xi^{\prime \prime}(s) \circ \xi^{\prime \prime}(t)^{-1} \circ \phi,
$$

belonging to $\Gamma\left(X^{\prime},[t, T]\right)$, is homotopic to $\xi^{\prime}$ at $t$. Thus by lemmas 7 and 24 , there exists a pointed section over $[0, T]$, contradiction.

II.3. Applications. We show how the criteria established in the preceding subsection apply in several typical situations.

Corollary 31. A surjective smooth submersion with each fibre diffeomorphic to $\mathbf{R}^{p}$ is a bundle.

Proof. The family VD of all vertical domains diffeomorphic to the compact $p$-ball is exhaustive, since each fibre is diffeomorphic to $\mathbf{R}^{p}$.

This family is obviously isotopy invariant.

Recall Alexander's trick: Let $M^{p}$ be any smooth manifold without boundary, let $E\left(\mathbf{B}^{p}, M\right)$ be the space of embeddings of the compact $p$-ball into $M$, and let $F(M)$ be the space of frames of $T M$. Then the map

$$
J_{0}^{1}: E\left(\mathbf{B}^{p}, M\right) \rightarrow F(M)
$$

that to each embedding associates its 1-jet at the origin, is a homotopy equivalence. 
It follows that for every $X, X^{\prime} \in \mathrm{VD}$ such that $X \subset \operatorname{Int}\left(X^{\prime}\right)$, the restriction map $\rho_{X, X^{\prime}}$ has contractible fibres. By lemma 8 it is a fibration. Thus theorem $\mathrm{B}$ applies.

Corollary 32. Let $\pi: E^{p+q} \rightarrow B^{q}$ be a (surjective, smooth) submersion-fibration.

a) If $p=2$, then $\pi$ is a bundle.

b) If $p \geq 5$, if each fibre $E_{b}$ is topologically finite, i.e. diffeomorphic to the interior of a compact $p$-manifold $M_{b}$ with smooth boundary $\partial M_{b}$, and if every connected component of every $\partial M_{b}$ is simply connected, then $\pi$ is a bundle.

c) (Stabilization) The map $E \times \mathbf{R}^{3 p+1} \rightarrow B:\left(x, x^{\prime}\right) \mapsto \pi(x)$ is a bundle.

Point c) answers a question of [3].

Proof. a) We can assume that the fibres are connected. Then the family of all connected vertical domains is obviously exhaustive and isotopy invariant. The engulfing property is satisfied by connected domains in surfaces - this is an exercise, using for example the results and methods of [2. Thus corollary 30 applies.

b) The set of all vertical domains $X \subset E_{b}$ such that $X$ is a deformation retract of $E_{b}$ is obviously exhaustive and isotopy invariant. By Van Kampen's and GrushkoNeumann's theorem, each component of $W=E_{b} \backslash \operatorname{Int}(X)$ is simply connected. By Poincaré duality and the $h$-cobordism theorem, $W \cong \partial X \times[0,1$ ( . The relative engulfing property follows immediately, and corollary 30 applies.

c) Given a domain $X$ and a polytope $K \subset \operatorname{Int}(X)$, say that $X$ shrinks to $K$ if, for every neighborhood $U(K)$, the whole domain $X$ can be pushed into $U(K)$ by an istopy of embeddings of $X$ into $X$, relative to a neighborhood of $K$.

For every $b \in B$, define the set $\mathrm{VD}_{b}$ of $(4 p+1)$-dimensional vertical domains $X \subset E_{b}^{\prime}=E_{b} \times \mathbf{R}^{3 p+1}$ with the two following properties

1. $X$ shrinks to a $(p-1)$-dimensional polytope.

2. For every $(p-1)$-dimensional polytope $K \subset \operatorname{Int}(X)$ there is a $(2 p-1)$ dimensional polytope $L \subset \operatorname{Int}(X)$ such that $K \subset L$ and such that $X$ shrinks to $L$.

Obviously VD is isotopy invariant. To prove that it is also engulfing, let $X, X^{\prime}$, $X^{\prime \prime} \in \mathrm{VD}_{b}$ be as in definition 29. Let $K, K^{\prime}$ be $(p-1)$-dimensional polytopes on which $X, X^{\prime}$ respectively shrink (property 1 ). Since $\operatorname{dim} K+\operatorname{dim} K^{\prime}<\operatorname{dim} E_{b}^{\prime}$, by a general position argument, we can chose $K, K^{\prime}$ disjoint. Since $X^{\prime} \in \mathrm{VD}$, by property 2 there is a $(2 p-1)$-dimensional complex $L \subset \operatorname{Int}\left(X^{\prime}\right)$ containing $K \cup K^{\prime}$ and to which $X^{\prime}$ shrinks.

By hypothesis, there is a homotopy that pushes $X^{\prime}$ into $X^{\prime \prime}$ and is the identity on $X$. Again by a general position argument, since $2(\operatorname{dim} L+1)<\operatorname{dim} E_{b}^{\prime}$, after a small perturbation if necessary, this homotopy induces, in restriction to $L$, an isotopy of embeddings of $L$ into $E_{b}^{\prime}$, and remains the identity on a neighborhood of $K$.

Since $X^{\prime}$ shrinks to $L$, we get an isotopy of embeddings of $X^{\prime}$ into $E_{b}^{\prime}$, that pushes it into $X^{\prime \prime}$. Moreover, it is the identity on a neighborhood of $K$.

Since $X$ shrinks to $K$, we can choose this isotopy to be identity on the whole of $X$, and the engulfing condition is established.

Finally, we prove that VD is exhaustive. It is enough to prove that for every domain $D^{p} \subset E_{b}$, the product $D^{p} \times \mathbf{B}^{3 p+1}$ is contained in a domain $X \in \mathrm{VD}$. Let $X$ be a regular neighborhood of $D^{p} \times \mathbf{B}^{3 p+1}$. In particular, $X$ shrinks to $D^{p} \times \mathbf{B}^{3 p+1}$. 
If $E_{b}$ is compact, then, since $\pi$ is assumed a fibration, all fibres are compact with the same $\pi_{0}$; thus, as previously mentionned, $\pi$ is already a bundle.

Thus we can assume that $E_{b}$ is not compact. Therefore $\partial D^{p}$ is not empty, and in particular $D^{p}$ shrinks to some $(p-1)$-subpolytope $K$. It follows obviously that $X$ also shrinks to $K$.

Finally, let $K \subset \operatorname{Int}(X)$ be any ( $p-1)$-polytope. We have to find a larger, $(2 p-1)$ dimensional polytope $L$ to which $X$ shrinks. After a first isotopy if necessary, we can assume that $K$ is contained in $D^{p} \times \mathbf{B}^{3 p+1}$. Decompose $E_{b}^{\prime}=E_{b} \times \mathbf{R}^{3 p+1}$ as $E_{b} \times \mathbf{R}^{p-1} \times \mathbf{R}^{2 p+2}$, and consider the canonical projection of $K$ into the factor $E_{b} \times \mathbf{R}^{p-1}$. Since $2 \operatorname{dim} K<2 p-1$, after a small perturbation this projection become an embedding of $K$ into $D^{p} \times \mathbf{B}^{p-1}$. Thus we have an isotopy of embeddings pushing $K$ into $D^{p} \times \mathbf{B}^{p-1}$. Thus we can assume that $K \subset D^{p} \times \mathbf{B}^{p-1}$. Then $L=D^{p} \times \mathbf{B}^{p-1}$ fits. In conclusion VD is exhaustive.

Thus corollary 30 applies.

Question. Assume that $\pi: E \rightarrow B$ is a submersion-fibration and that a group acts freely, cocompactly and properly discontinuously on $E$ and permutes the fibres. Does it follow that $\pi$ is a bundle?

As a last application, in corollary $31, \mathbf{R}^{p}$ can be replaced by any manifold bounded by the sphere and of large enough dimension.

Corollary 33. Let $M$ be a compact manifold of dimension $p \geq 5$ and with smooth boundary homeomorphic to $\mathbf{S}^{p-1}$. Then any (surjective, smooth) submersion all of whose fibres are diffeomorphic to $\operatorname{Int}(M)$, is a bundle.

Observe that here we no longer assume the submersion to be a fibration.

Proof. We first claim that for every submanifold $X \subset \operatorname{Int}(M)$ abstractly diffeomorphic with $M$, the pair $(M, X)$ is contractible.

Indeed, after Van Kampen's and Grushko-Neumann's theorems, $W$ is simply connected. Also, the Mayer-Vietoris sequence for $M=X \cup W$ gives, for each $2 \leq k \leq p-2$, that $H^{k}(X ; \mathbf{Z}) \oplus H^{k}(W ; \mathbf{Z})$ is isomorphic to $H^{k}(M ; \mathbf{Z})$. Since $H^{k}(X ; \mathbf{Z})$ is (abstractly) isomorphic to $H^{k}(M ; \mathbf{Z})$, it follows from the elementary theory of finitely generated abelian groups that $W$ is $(p-2)$-connected. Thus the pair $(W, \partial X)$ is $(p-2)$-connected. On the other hand, by Poincaré duality, $H_{k}(W, \partial X ; \mathbf{Z})$ is isomorphic to $H^{n-k}(W, \partial M ; \mathbf{Z})$, thus null for $k \geq n-1$ since $W$ is 1 -connected and $\partial M$ is 2 -connected.

To sum up, $(W, \partial X)$ has no homology. By the excision principle, $(M, X)$ doesn't either. The claim is proved.

Fix $b \in B$. Choose a core $X \subset E_{b}$. By lemma 24, there is a neighborhood $Y$ of $b$ in $B$ and a section $\gamma \in \Gamma(X, B)$. By the claim, for every $y \in Y$, the vertical domain $\gamma(y)(X)=\operatorname{Im} \gamma \cup E_{y}$ is a deformation retract of $E_{y}$. By corollary 15, $\pi$ is a fibration over $Y$. Corollary 32, b) now yields the desired conclusion.

It follows that Palmeira's conjugation theorem for open manifolds foliated by $\mathbf{R}^{p}$ extends to more general leaves; the (long) proof is exactly as in 9].

Corollary 34. Let $M^{p}$ be as in the preceding corollary. Let $(V, F)$ and $\left(V^{\prime}, F^{\prime}\right)$ be foliated, simply connected, $(p+1)$-dimensional, open manifolds whose leaves are all diffeomorphic to $M$. Assume that the (in general non-Hausdorff) leaf spaces $V / F$ and $V^{\prime} / F^{\prime}$ are diffeomorphic. Then this diffeomorphism can be realized by a smooth conjugation between the foliations. 
II.4. Ends of deformation-equivalent manifolds. What must two different fibres $E_{b}, E_{b^{\prime}}$ of a fibration-submersion $\pi: E \rightarrow B$ have in common, assuming of course that $B$ is connected?

Call two such open manifolds, say, deformation-equivalent. Compare [3].

Of course they have the same homotopy type, but not necessarily the same proper homotopy type (example 21). In fact, every contractible $p$-manifold $U$ is deformation-equivalent to $\mathbf{R}^{p}$ : proceed as in example 21 , but change $\mathbf{R}^{p}$ to $U$, and $W$ to a small open $p$-ball in $U$.

Nevertheless, in dimension 2, deformation-equivalent manifolds are necessarily diffeomorphic (corollary 32).

The following proposition answers a question of [3], where some particular cases were obtained.

Proposition 35. Two deformation-equivalent manifolds necessarily : a) are diffeomorphic if one of them is compact; b) have the same tangential homotopy type; c) have the same orientability property w.r.t. every ring $R$; d) have isomorphic algebras of compactly supported cohomology with coefficients in every ring $R$ that makes them orientable; and e) have homeomorphic ends spaces.

Proof. a) If $E_{b}$ is compact, then all fibres having the same dimension and homotopy type as $E_{b}$ are compact; moreover, they have the same $\pi_{0}$, and we have seen as a corollary of lemma 22 that $\pi$ is necessarily a bundle.

b) holds because the tangent vector spaces $T E_{b}$ and $T E_{b^{\prime}}$ are two pullbacks of a single $p$-dimensionnal vector space over $E$ : the kernel of $d \pi$.

c) follows from b).

d) and e) One can assume that $B=\mathbf{B}^{q}$ and that $b, b^{\prime} \in \operatorname{Int}(B)$. Thus $E$ has a boundary $\partial E=\pi^{-1}(\partial B)$. We shall prove that $E_{b}$ has the same cohomology algebra and the same ends space as $E$; of course the same will be true for $E_{b^{\prime}}$.

We have a commutative diagram

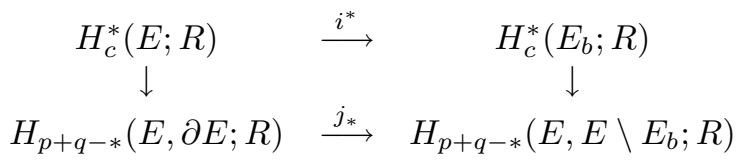

The vertical arrows are Poincaré and Alexander duality, and they are one-to-one; $i^{*}$ is the morphism of restriction to the properly embedded submanifold $E_{b}$; and $j_{*}$ is the inclusion morphism, one-to-one because $\partial B$ is a deformation retract of $B \backslash\{b\}$ and because $\pi$ is a fibration. Thus $i^{*}$ is one-to-one, and at the same time an algebra morphism. This proves d).

For every locally compact space $S$, its space of ends can be defined as the spectrum of an algebra, namely the algebra $B(S)$ of germs, in the neighborhood of infinity, of locally constant functions with value in $R=\mathbf{Z} / 2 \mathbf{Z}$. On the other hand, we have a commutative diagram whose rows are exact:

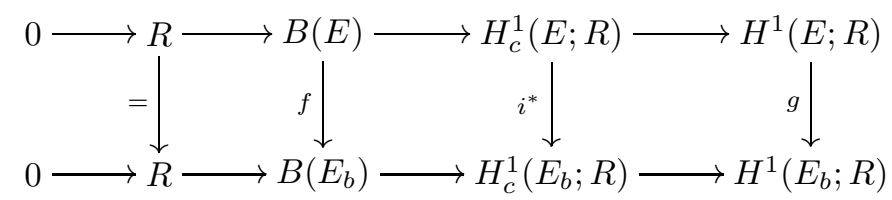


where $f, i^{*}$ and $g$ are restriction morphisms. Since $\pi$ is a fibration, $g$ is one-to-one. By d), $i^{*}$ is one-to-one. By the five lemma, $f$ is also one-to-one. Thus the algebras $B(E)$ and $B\left(E_{b}\right)$ are isomorphic; hence they have homeomorphic spectra.

II.5. More examples and questions. We end with a few (pleasant) monsters.

Example 36. Let $W$ again be the Whitehead manifold, let $V \subset W$ be an open 3-ball, and let $U \subset V$ be an open subset diffeomorphic to $W$. Play the same game as in example 21, but with $W$ instead of $\mathbf{R}^{3}$ and with $U$ instead of $W$. Then again $\pi$ is a fibration of $\mathbf{R}^{4}$ onto $\mathbf{R}$, but this time all fibres are diffeomorphic to $W$. If it were a bundle, then there would be a 1-parameter family of embeddings $i_{t}: W \rightarrow W$ such that $i_{0}=\operatorname{id}_{W}$ and $i_{1}(W)=U$. But this would imply that every compact subset of $W$ could be engulfed by the 3-ball $V$, and $W$ would be diffeomorphic to $\mathbf{R}^{3}$, a contradiction. Thus $\pi$ is not a bundle.

Example 37. Let $V \subset \mathbf{R}^{4}$ be an open subset homeomorphic but not diffeomorphic to $\mathbf{R}^{4}$ (Casson-Friedman, see for example [6]). Play the same game as in example 21 , but with $\mathbf{R}^{4}$ instead of $\mathbf{R}^{3}$ and with $V$ instead of $W$. The total space $E$ is diffeomorphic to $\mathbf{R}^{5}$ since it is 5-dimensional, contractible and simply connected at infinity [13]. This (real-analytic!) submersion-fibration of $\mathbf{R}^{5}$ onto $\mathbf{R}$ is not a bundle in the $C^{\infty}$ (or even $C^{1}$ ) category, since one of the fibres is not diffeomorphic to the others. On the other hand, all our work extends to the $C^{r}$ categories $(r \in \mathbf{N})$. The reader will provide a proof of lemma 22 in the $C^{0}$ and the $C^{1}$ differentiability class. In particular, corollary 31 is valid in class $C^{0}$; thus our submersion-fibration is a bundle in the $C^{0}$ sense.

Example 38. Let

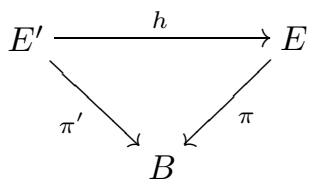

be a commutative diagram of (smooth, surjective) submersions. If $\pi^{\prime}$ and $h$ are fibrations, so is $\pi$; this is obvious, since to be a fibration it is enough to have the homotopy lifting property for simplicies. Jean Pradines asked if we can change "fibration" to "bundle". The answer is negative, always with the same counterexample. Let $E, B, \pi$ be as in example 21 , let $E^{\prime}=E \times \mathbf{R}$ and let $h$ be the first projection. Then $h$ is a bundle. Also $\pi^{\prime}$ is a bundle by corollary 31 , since all its fibres are $\mathbf{R}^{3} \times \mathbf{R}$ or $W \times \mathbf{R}$, thus diffeomorphic to $\mathbf{R}^{4}$. But $\pi$ is not. This phenomenon was already observed in [3].

Example 39. We can also answer Pradines' question negatively with $h$ a normal, infinite cyclic covering. Let $E^{*}, B, \pi^{*}$ be as in example 21 , let $E=E^{*} \times \mathbf{S}^{1}$, let $\pi(x, y)=\pi^{*}(x)$, let $E^{\prime}$ be the universal covering of $E$ and let $h: E^{\prime} \rightarrow E$ be the canonical projection. Then $h$ is a normal covering, and again $\pi^{\prime}$ is a bundle by corollary 31 , since $W \times \mathbf{R}$ is diffeomorphic to $\mathbf{R}^{4}$. But $\pi$ is not, since $W \times \mathbf{S}^{1}$ does not have the same proper homotopy type as $\mathbf{R}^{3} \times \mathbf{S}^{1}$.

Example 40. As Alan Weinstein points out [14], our results also allow us to answer Pradines' question negatively with $E$ the quotient of $E^{\prime}$ by the free, fibrewise action of a compact group. Actually, let $E, B, \pi$ be as in example 37 (thus with a fibre being an exotic $\mathbf{R}^{4}$ ), let $E^{\prime}=E \times \mathbf{S}^{3}$ and let $h$ be the first projection. Then $h$ is 
a bundle. Also, each fibre of $\pi^{\prime}$ is $\mathbf{S}^{3} \times$ a topological $\mathbf{R}^{4}$, and thus the interior of a 7-dimensional compact manifold with (simply connected) boundary, by [12]. By corollary $32 \mathrm{~b}$ ), $\pi^{\prime}$ is a bundle. But $\pi$ is not.

Question (Weinstein). What about Pradines' question with $h$ a finite covering? Or equivalently, if one prefers, a normal one, i.e. the quotient by the free fibrewise action of a finite group?

Some of these results have been announced or conjectured in [7] and [8. One can find applications to flows and foliations in 9 .

\section{REFERENCES}

[1] Cerf J., Topologie de certains espaces de plongements, Bull. Soc. Math. France 89 (1961), 227-380. MR 25:3543]

[2] Epstein D.B.A., Curves on 2-manifolds and isotopies, Acta Mathematica 115 (1966), 83-107. MR 35:4938

[3] Ferry S., Alexander duality and Hurewicz fibrations, Trans. Amer. Math. Soc. 327, 1 (1991), 201-219. MR 91m:55015]

[4] Haefliger A., Groupoïde d'holonomie et classifiants. in Structures transverses des feuilletages. Toulouse 1982, Astérisque 116 (1984), 70-97. MR 86c:57026a

[5] Hermann R., A sufficient condition that a mapping of Riemannian manifolds be a fiber bundle. Proc. Amer. Math. Soc. 11 (1960), 236-242. MR 22:3006

[6] Kirby R.C., The topology of 4-manifolds, Springer Lecture Notes in Mathematics 1374 (1989). MR 90j:57012

[7] Meigniez G., Submersions et fibrations localement triviales. C. R. Acad. Sci. Paris, 321, série I (1995), 1363-1365. MR 96m:57054

[8] Meigniez G., Sur le relèvement des homotopies. C. R. Acad. Sci. Paris, 321, série I (1995), 1497-1500. MR 97g:57008

[9] Palmeira C.F.B., Open manifolds foliated by planes, Ann. of Math. 107 (1978), 109-131. MR 58:18490

[10] Meigniez, G. Prolongement des homotopies, Q-variétés et cycles tangents, Ann. Inst. Fourier, Grenoble 47, 3 (1997), 945-965. MR 98h:57052

[11] Reinhart B.L., Foliated manifolds with bundle-like metrics, Ann. of Math. 69, 1 (1959), 119132. MR 21:6004

[12] Siebenmann L., Thesis, Princeton U. (1965). See http://www.maths.ed.ac.uk/people/aar/ surgery/sieben.poly

[13] Stallings J., The piecewise linear structure of euclidian space, Proc. Cambridge Philos. Soc. 58 (1961), 481-488. MR 26:6945

[14] Weinstein A., Linearization of regular proper groupoids, preprint, Berkeley (2001). To appear in J. Inst. Math. Jussieu.

Laboratoire de Mathématiques et D'Application des Mathématiques, Université de Bretagne Sud, Campus de Tohannic, Centre de recherche, F- 56017 Vannes Cedex, France

E-mail address: Gael.Meigniez@univ-ubs.fr 